\title{
Four Strategies for Finding Good Evidence Efficiently
}

\author{
Mark P. MacEachern, MLIS ${ }^{1}$
}

Searching PubMed and other citation resources is an essential component of evidencebased practice. The inability to create effective searches can impact the ability of clinicians to incorporate evidence into their decision-making processes. This paper describes four strategies to help clinicians find evidence efficiently through targeted, well-designed literature searches. Int J Evid Based Pract Dent Hygienist 2016;2:XX-XX. doi: 10.11607/ebh.59

Keywords: [Author: Please provide a few keywords]

${ }^{1}$ Informationist, Taubman Health Sciences Library, University of Michigan, Ann Arbor, Michigan, USA.

\section{Correspondence to:}

Mark P. MacEachern

Taubman Health Sciences Library

University of Michigan

1135 Catherine Street

Ann Arbor, MI 48109, USA

[AU: Is this mailing address correct?]

Email:markmac@umich.edu

C2016 by Quintessence

Publishing Co Inc.

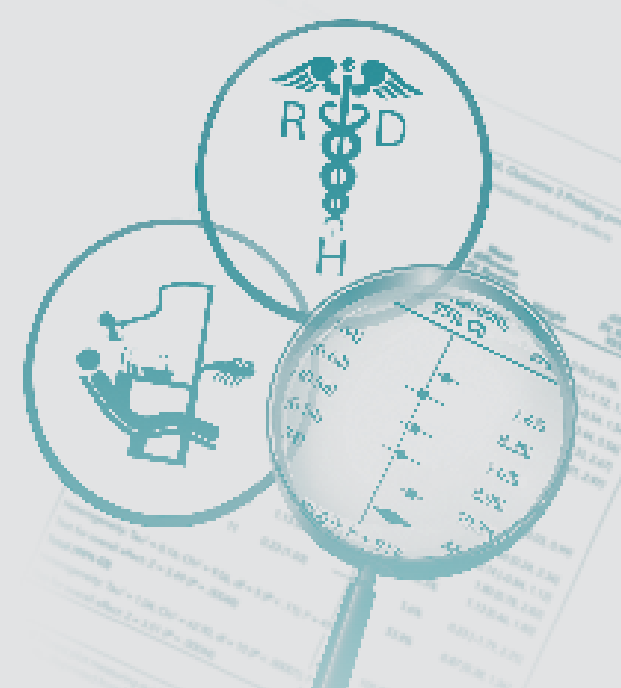

If you've searched PubMed before, you have likely encountered results that are too extensive to navigate efficiently. The same can be said of Google and Google Scholar. Both have their place in evidence-based practice, but search results can become unwieldy with the thousands of articles and seemingly never-ending supply of journals that publish them. To make matters worse, it can be difficult to distinguish the quality studies from those at the other end of the spectrum. How to navigate this mass of citations is a common predicament for those attempting to incorporate best evidence into their practice. Fortunately, there are resources and strategies that can facilitate the literature search process.

Through my years as a literature searcher and educator in the health sciences, I have found that efficient searching is aided by four strategies:

1. Break your topic into searchable concepts.

2. Use ANDs and ORs correctly.

3. Know when to target primary and secondary literature.

4. Make the resource work for you.

Clinicians who adopt these strategies will be better equipped to navigate the ever-expanding corpus of evidence.

Each of these strategies is explained below, but first let's consider the resources that will be used to frame the discussion.

\section{PubMed and Google Scholar}

PubMed and Google Scholar are online gateways to the scientific literature-the type of literature that underpins evidence-based practice. PubMed (http://www.ncbi.nlm.nih.gov/pubmed) is a free citation resource, maintained by a group within the United States National Institutes of Health, that consists of nearly 26 million citations as of this writing. The citations are predominately from health sciences journals, including those representing 


\section{BOX 1 PICO-formatted question}

$$
\begin{aligned}
& \text { P-Gag reflex } \\
& \text { I - Acupuncture } \\
& \text { C - None } \\
& \text { O- Reduction in gag response }
\end{aligned}
$$

PICO-formatted question:

In dental patients with gag reflex, is acupuncture (compared with nothing) an effective strategy to reduce gag response?

dentistry and dental hygiene. PubMed is a major resource for clinicians attempting to find the best studies to address specific clinical questions.

Google Scholar (http://scholar.google.com), like PubMed, is a free-to-search resource that provides access to millions of citations. While Google searches the web, Google Scholar searches the scholarly literature. It is interdisciplinary, consisting of, but not limited to, the health sciences journal literature. While there is overlap in content between PubMed and Google Scholar, the two resources complement each other well.

Clinicians can search both of these resources to find evidence to support their clinical decisions and keep up to date with the journal literature. There are other free-to-search resources, including the American Dental Association's Center for Evidence-Based Dentistry site (http://ebd.ada.org), the National Guidelines Clearinghouse (http://www.guidelines. gov), and the TRIP Database (http://www.tripdatabase.com), which are great sources of evidence but not the focus of this article.

Now, let's take a look at each strategy in more detail.

\section{Break your topic into searchable concepts}

To search resources effectively, clinicians should think of clinical concerns in terms of concepts. When approaching a literature search, I always think with the end in mind and ask myself, what concepts do I want to see represented in a relevant study? If your patient is exhibiting a gag reflex that makes dental treatment difficult and you are interested in the evidence supporting various management strategies, then a relevant study will be about patients with gag reflexes in the context of dentistry, and your search will combine variations of those two concepts: gag reflex and dentistry. Or, gag reflex and management. Rarely is it preferable to search in complete sentences.

$\mathrm{PICO}$-acronym for patient, intervention, comparison, outcome-is a popular model of question formulation in evidence-based practice to help clinicians identify key concepts and frame those concepts in an answerable question that can be searched in literature resources. There are many tutorials online that walk clinicians through the PICO process, which you can find by combining PICO and EBD in a Google search.

Using the above scenario, the patient $(P)$ would be an individual with gag reflex. We could be more specific about the individual if, for instance, sex or age were relevant to the question. Otherwise, gag reflex is sufficient. Keep in mind that the more specific we are, the fewer results we will get. This could be problematic if too few results are obtained.

The intervention (I) represents the action we are taking to address whatever clinical issue is of concern to the patient. In our scenario, the intervention could be a specific management strategy such as acupuncture or management strategies in general. In the case of the former, the I would be acupuncture. In the latter, it would be management.

The comparison (C) is the action with which our intervention is being compared. In this case, the C could be another intervention, such as laser therapy, or it could be left blank if we do not have a comparison.

The outcome $(\mathrm{O})$ is the goal of the intervention, so in this case an improvement or reduction in gag response. When searching PubMed or Google Scholar, you can often build a search around the $P$ and $I$ and leave out the $C$ and $O$ entirely to avoid getting too few results.

The terms we use in PICO can then be translated into an answerable clinical question, as shown in Box 1 , and those terms would serve as a basis for the literature search.

\section{Use ANDs and ORs effectively}

The next step is to structure an effective search around the key concepts you just identified. The Boolean operators AND and OR are an important part of this. They represent the fundamental logic that underlies many search interfaces and are used to define the relationship between concepts. AND is used to combine distinct concepts, while OR is used to combine synonymous concepts (Fig 1). 
In our scenario, we have two distinct concepts, gag reflex and dentistry, so the Boolean AND is the appropriate operator to define their relationship (eg, gag reflex AND dentistry). By combining gag reflex and dentistry with AND, we are informing the resource that we are interested in citations that have both concepts-the intersection of the overlapping circles in Fig 1.

AND is often implied between terms in search interfaces and thus unnecessary at times, but it is still possible to use the operator incorrectly. For example, in PubMed, the search gag reflex AND dentistry is equivalent to gag reflex dentistry (with no AND). But it is not equivalent to gag AND reflex AND dentistry. The latter is an outlier because the first AND in that search disrupts PubMed's attempt to search for gag reflex as one concept. In the other two examples, PubMed intelligently recognizes that gag reflex is one concept and dentistry is another.

We can expand upon this search by incorporating synonyms. Giving thought to synonyms is important because more than one term or phrase may be used to describe the same concept. For example, some authors will use gagging or pharyngeal reflex to represent the concept of gag reflex. By relying solely on gag reflex in our search, we may miss studies that use synonymous terms or phrases, and missed studies in an evidence-based practice context can impact our clinical decisions.

To build these variations into our search, we use the operator OR. Such a search would look like this:

\section{(gag reflex OR gagging OR pharyngeal reflex) AND dentistry}

When searching PubMed and other resources it is imperative that the terms connected with the operator OR are grouped or nested together in parentheses. The use of parentheses tells the database that those terms should be considered one concept, and the database will then return citations that have at least one of those terms.

We can do the same for the dentistry concept. Or, as a change of approach, we could connect OR with specific interventions for gag reflex, such as acupuncture, acupressure, laser therapy, and behavioral therapy. We would use these concepts in our search if we were interested in studies that pertained to those specific interventions for gag reflex. Our modified search would look like this:

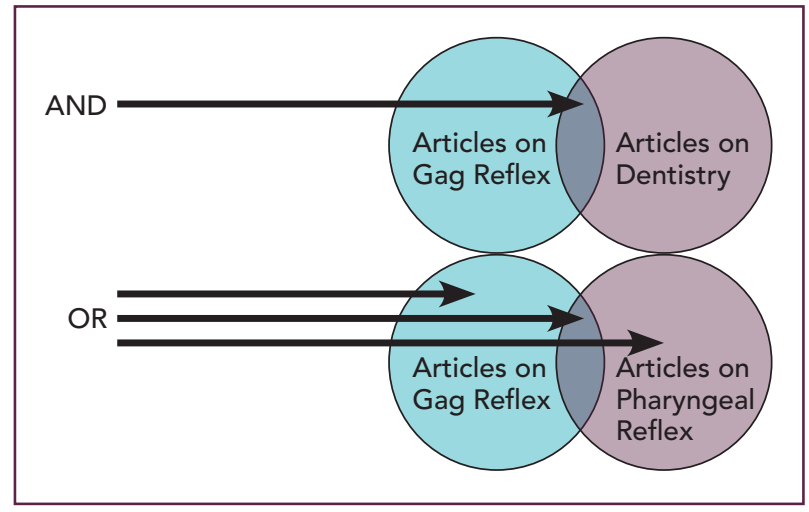

Fig 1 AND and OR. AND captures articles that have both concepts. OR captures articles that have either concept.

(gag reflex OR gagging OR pharyngeal reflex) AND (acupuncture OR acupressure OR laser OR lasers OR behavioral OR behavioural)

If we fail to include the parentheses, most resources will only use the Boolean operator in conjunction with the word immediately preceding and following it. In other words, the AND will only apply to pharyngeal reflex and acupuncture. All the other words would be rogue, on their own, untouched by any AND, which would inflate the number of results. You might not need to use OR very often in your clinical searches, but when you do it is important to use it correctly, which frequently requires parentheses.

Note that in some resources you can use an asterisk to pick up word endings. Consider the following example:

\section{(gag reflex* OR gagging OR pharyngeal reflex*) AND (acupuncture* OR acupressure* OR laser* OR behavi*)}

The asterisk on gag reflex* picks up gag reflex, gag reflexes, and other endings. Similarly, behavi* picks up behavior, behaviors, behavioral, and British equivalents (-iour).

\section{Know when to target primary and secondary literature}

The first two practices focus on the construction of effective search strategies. The third focuses on the results, specifically the ability to target the most appropriate type of study. Not all study types are 


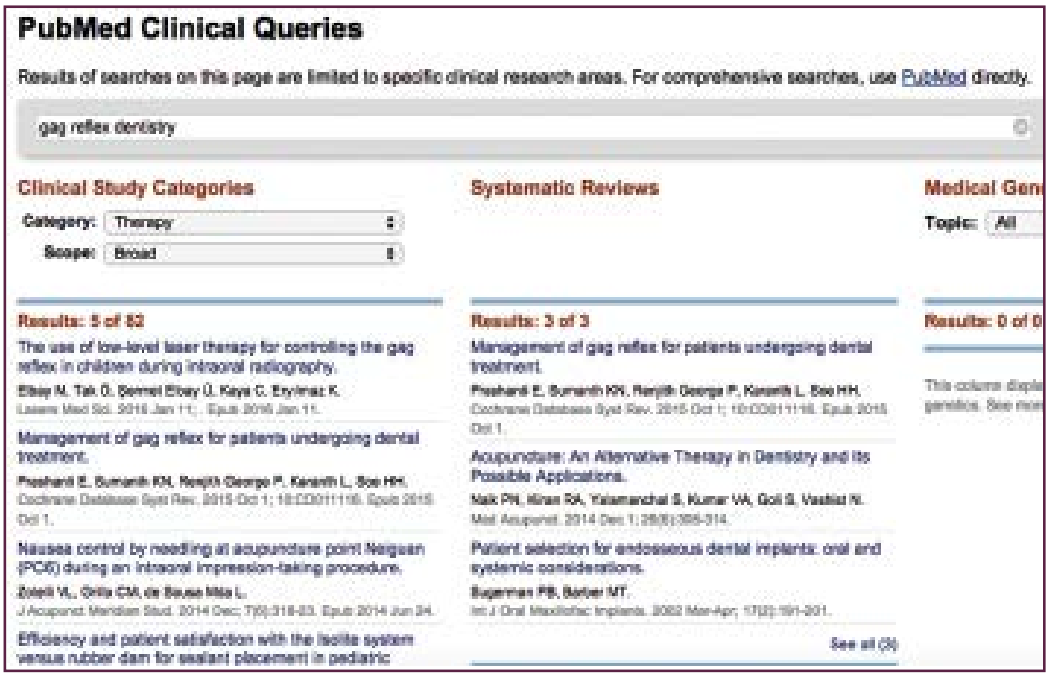

Fig 2 PubMed Clinical Queries. The Clinical Study Categories column on the left targets primary studies. The Systematic Reviews column targets systematic reviews and other types of reviews. suitable for addressing certain clinical questions. If you know the type of study to target before you start running searches, you can save a lot of time and more easily find the best evidence. An understanding of primary and secondary literature helps with this process.

Primary literature in the health sciences represents original research. In evidence-based practice, this usually means clinical studies. Consider the following scenario: you are working in a dental clinic and conducting a study on whether one type of fluoride treatment is better than another at preventing dental caries. You and the other members of the research team use one type of fluoride on one group of patients, and another type on another group of patients. You track the patients' dental health over a period of time and ultimately conclude that one type of fluoride is better than the other. You and the team write up the study's results and submit the paper to a dental hygiene journal. The published paper is an example of primary literature; it is the publication closest to the data.

Secondary literature describes articles that are built on the primary literature. Systematic reviews are a good example because their conclusions are based on a pooled collection of original research studies. Imagine that three other dental clinics around the country conducted similar studies comparing one type of fluoride with another and published their results in various journals. Each of these studies individually is best described as primary literature; however, when the four studies are grouped together in a review paper, the resulting publication would fall under the umbrella of secondary literature.

The results of the four studies pooled together in a review paper should be more meaningful than those of any one study. In evidence-based practice, then, it is ideal to target that review paper first and only venture to the primary literature if your search fails. With that said, not all review papers should be weighted equally in your decision-making. Systematic reviews, meta-analyses, evidence-based summaries, practice guidelines, and other reviews that are compiled with an evidence-based methodology are excellent decision aids if they are relevant to your topic. Reviews that do not use a clear methodology are less impactful because, for one, the articles selected and discussed are not included for any discernably objective reason. Even systematic reviews can be poorly designed and suffer the same limitations as other types of studies. As a result, it is important for clinicians to consider the quality of all studies, including systematic reviews, before treating their conclusions and recommendations as "truth."

There are times when you will need to delve into the primary literature. These searches can be more time-consuming simply because there are more primary studies, and it is impractical to read through dozens of relatively similar primary studies to find the one best suited to your question. Common study types include randomized controlled trials (RCTs), cohort studies, case series, and case reports. 
All have their strengths. All have their limitations. All are best suited for certain types of questions. RCTs are great for treatment questions, while a case report, which is simply an article about a clinical case that is noteworthy for some reason, might be the best information you can find about a novel diagnosis or treatment strategy.

Many resources have filters that clinicians can use to target specific types of studies. PubMed, for example, has Clinical Queries, which easily allows clinicians to see primary studies and systematic reviews isolated on one screen. A clinician can scan the Systematic Reviews column first, and if the results are lacking, then immediately jump to the Clinical Study Categories column for relevant primary studies (Fig 2).

By targeting a specific type of study, you are applying parameters to your search that will reduce the number of citations you see, which will save you time and help bring the most relevant studies to the forefront of your search results.

\section{Make the resource work for you}

A major challenge for clinicians who practice in a thoughtful, evidence-based manner is finding the time to do it effectively. Making use of the alerting features available in many resources can help in this regard. Most resources allow you to create accounts and set up alerts to receive new citations on research or clinical topics as well as journal tables of contents of interest to you. For example, if you wish to keep up on the gag reflex literature, you can access PubMed, create a relatively comprehensive search to capture the literature on the topic, set up an alert, and then receive emails as new gag refex citations become available. By taking advantage of these alerting features, you are effectively creating a system in which the resources work for you by sending targeted literature directly to you (Box 2).

\section{BOX 2 How to set up an alert in PubMed}

1. Create a search in PubMed.

2. Click Create Alert.

3. Create an account with NCBI by following the instructions.

4. Name the search and decide on an alerting schedule. Click Save.

5. Start receiving updates when new citations that meet your search criteria become available in PubMed.

\section{Conclusions}

Each resource functions in its own particular way, but the four strategies presented in this article form a solid foundation for getting the best results from the resources you will use in searching for evidence. By adopting these practices, your searches will be facilitated and you will be better equipped to adopt evidence-based practice.

\section{Acknowledgments}

The author reports no conflicts of interest. 